\title{
Applanation Tonometry
}

National Cancer Institute

\section{Source}

National Cancer Institute. Applanation Tonometry. NCI Thesaurus. Code C120682.

A method in which a constant pressure is applied to the surface of the cornea in order to

flatten a prespecified area of the membrane, which is used to calculate intraocular pressure. 